\title{
How different is the care of terminal pancreatic cancer patients in inpatient palliative care units and acute hospital wards? A nationwide population-based study
}

Jack P. Wang ${ }^{1,2,3}$, Chen-Yi Wu ${ }^{4,5^{*}}$, I-Hsuan Hwang $^{6}$, Chien-Hui Kao ${ }^{1,2}$, Yi-Ping Hung ${ }^{1,2,7}$, Shinn-Jang Hwang ${ }^{2,8}$ and Chung-Pin $\mathrm{Li}^{1,2^{*}}$

\begin{abstract}
Background: Inpatient palliative care is important for patients with terminal pancreatic cancer. However, the differences between inpatient palliative care and acute hospital care for inpatients with pancreatic cancer have not been explored in a population-based study.

Methods: This population-based nationwide study was conducted using data from the Taiwan National Health Insurance database to analyze the differences between inpatient palliative care and acute hospital care for inpatients with pancreatic cancer. We identified 854 patients with terminal pancreatic cancer, who had received in-hospital end-of-life care between January 2003 and December 2006. These patients were then sub-divided and matched 1:1 (using propensity score matching) according to whether they received inpatient palliative care $(n=276)$ or acute hospital care $(n=276)$. These groups were subsequently compared to evaluate any differences in the use of aggressive procedures, prescribed medications, and medical costs.

Results: Inpatient palliative care was typically provided by family physicians (39\%) and oncologists (25\%), while acute hospital care was typically provided by oncologists (29\%) and gastroenterologists (24\%). The inpatient palliative care group used natural opium alkaloids significantly more frequently than the acute hospital care group ( $84.4 \%$ vs. $56.5 \%$, respectively; $P<0.001$ ). The inpatient palliative care group also had shorter hospital stays $(10.6 \pm 11.1$ days vs. $20.6 \pm 16.3$ days, respectively; $P<0.001)$, fewer aggressive procedures, and lower medical costs (both, $P<0.005$ ).
\end{abstract}

Conclusions: Compared to patients in acute hospital wards, patients with pancreatic cancer in inpatient palliative care units received more frequent pain control treatments, underwent fewer aggressive procedures, and incurred lower medical costs. Therefore, inpatient palliative care should be considered a viable option for patients with terminal pancreatic cancer.

Keywords: End-of-life care, Pancreatic cancer, Hospice, Inpatient palliative care

\footnotetext{
*Correspondence: chenyiok@gmail.com; cpli@vghtpe.gov.tw

${ }^{4}$ Department of Dermatology, Taipei Veterans General Hospital, No. 201, Sec.

2, Shih-Pai Road, Taipei 11217, Taiwan

'Division of Gastroenterology and Hepatology, Department of Medicine,

Taipei Veterans General Hospital, No. 201, Sec. 2, Shih-Pai Road, Taipei 11217,

Taiwan

Full list of author information is available at the end of the article
} 


\section{Background}

Pancreatic cancer is one of the worldwide leading causes of cancer-related death [1], and pancreatic cancer is the fourth most common type of solid tumor in the United States [1]. Unfortunately, most patients with pancreatic cancer are in the advanced stages, and have a median expected survival period of $<8$ months [2]. In addition, patients with terminal pancreatic cancer frequently suffer from weight loss, jaundice, loss of appetite, nausea, vomiting, abdominal pain, back pain, cachexia, esophageal variceal bleeding, and ascites [2]. Therefore, symptomatic treatment is important for relieving the discomfort that is experienced by these patients.

Hospice care aims to provide supportive care to patients who are in the final stage of a terminal illness [3]. Supportive care is the treatment given to prevent, control, or relieve complications and side effects and to improve the patient's comfort and quality of life [4]. Therefore, hospice care focuses on improving the patient's comfort and quality of life, rather than achieving a cure for their condition. Hospice programs typically use a multidisciplinary approach, which includes the services of doctors, nurses, social workers, and clergy, in order to offer holistic care to patients. Based on this comprehensive care, it has been reported that patients who receive hospice care experience a better quality of life, compared to patients with similar conditions who receive conventional care [5].

Palliative care is an approach that improves the quality of life of patients and their families facing the problems that are associated with life-threatening illness, through the prevention and relief of suffering by early identification and impeccable assessment and treatment of pain and other physical, psychosocial, and spiritual problems [6]. Inpatient palliative care in Taiwan is covered by National Health Insurance (NHI) programs, and is offered by many medical centers (medical facilities that are larger than regional or district hospitals) or schoolaffiliated hospitals. However, only a small percentage of terminally ill patients ultimately select inpatient palliative care $[7,8]$, and the rate of inpatient palliative care use was $12.3 \%$ among patients with cancer who died between 2000 and 2004 [8]. Furthermore, although the trend of inpatient palliative care use increased from $5.5 \%$ in 2000 to $15.4 \%$ in 2004, the rate of Taiwanese inpatient palliative care use remains far below the rates in Western countries [7-10]. This relatively low usage may be due to individual misconceptions, physician preferences, and/or cultural concerns [9, 10]. A previous study in a Taiwanese inpatient palliative care unit reported that inpatients with advanced cancer in inpatient palliative care units had a shorter length of stay, compared to inpatients who were treated at acute hospital wards [11]. Nevertheless, there are no studies that have performed a comprehensive, nationwide comparison of inpatient palliative care and acute hospital care for patients with pancreatic cancer. Therefore, using information from Taiwan's NHI database, we aimed to compare the patient characteristics, medical procedures, prescriptions, and medical costs for patients with pancreatic cancer who received inpatient palliative care or acute hospital care, and to identify any significant differences between these groups.

\section{Methods}

\section{Data sources}

Taiwan's NHI program is a compulsory universal health insurance program (approximately $97 \%$ of all residents were enrolled in 2004), which offers comprehensive medical care coverage to all residents. The NHI dataset, which is compiled and managed by the National Health Research Institutes and publicly available to researchers, consists of comprehensive healthcare information from all NHI beneficiaries [12].

In this study, we obtained records, including medical order files and hospital administration files, from all inpatient beneficiaries who made a claim between January 2003 and December 2006. Using these records, we extracted anonymized inpatient identification numbers, dates of admission and discharge, 1 major and 4 minor disease ICD-9-CM diagnoses (International Classification of Diseases, 9th Revision, Clinical Modification), examination costs, procedures, surgeries, prescribed drugs, and special medical materials that were used during the admission. The patients' medical order files also contained details regarding the doctors' orders and laboratory tests. The accreditation level of each hospital (i.e., medical center, regional hospital, or district hospital) was determined using the hospital's administration files. A complete database of the coding numbers, which identifies the prescribed drugs and procedures, were obtained from the NHI website [13].

This study was conducted in accordance with the Helsinki Declaration, and was approved by the institutional review board of National Yang-Ming University (approval no. 1000046).

\section{Study population}

In this study, we identified 854 pancreatic cancer patients who were admitted and died in-hospital between January 2003 and December 2006. Pancreatic cancer was defined using an ICD-9-CM compatible code (157) from the Registry for Catastrophic Illness Patient database, which is a separate subsection of the NHI database. Most patients who are diagnosed with cancer sign up for a Catastrophic Illness Card in Taiwan, and these cardholders are exempt from cost-sharing under the NHI program. The effects of comorbidities were estimated 
using the Charlson comorbidity index [14]. Propensity score matching was performed to minimize the potential influence of selection bias, whereby logistic regression was used to create a propensity score for the inpatient palliative care and acute hospital care groups [15]. The covariates that we examined included patient characteristics (age and sex), Charlson comorbidity index, hospital location, and hospital accreditation level. One-to-one matching was subsequently performed for the two patients groups, using the nearest-neighbor matching method.

\section{Statistical analysis}

Data linkage, processing, and computation were performed using Microsoft Structured Query Language Server 2008 (Microsoft Corp., Redmond, WA, USA), and SPSS software (version 21.0, SPSS Inc., Chicago, IL, USA) was used to analyze the data for descriptive and inferential statistics. The Mann-Whitney $U$ test was used to analyze numerical data between the groups, and the chi-square test was used to compare categorical variables. A generalized linear model with gamma distribution and a log link function was used to analyze medical costs, which are typically right-skewed [16]. Differences were considered statistically significant at a $P$-value of $<0.05$. All data were presented as mean \pm standard deviation or number (\%) as appropriate.

\section{Results}

\section{Baseline characteristics}

Among the 854 terminally ill patients with pancreatic cancer that we identified, 276 (32 \%) patients were treated in inpatient palliative care units (173 men and 103 women; mean age $=68.6$ years $)$ and $578(68 \%)$ were treated at acute hospital wards (354 men and 224 women; mean age $=67.5$ years) (Table 1 ). All physicians who treated these patients had passed standardized hospice specialist training courses in Taiwan. The inpatient palliative care group was typically managed by family physicians (39\%) and oncologists (25\%), whereas the acute hospital care group was typically managed by internal medicine physicians (39\%, $P<0.001$ ), who were generally gastroenterologists $(24 \%)$. Significant differences between the palliative care and pre-matched acute care patients were observed for the hospital accreditation level $(P=0.03)$ and hospital regional location $(P=$ 0.021). The Charlson comorbidity indices were not significantly different between the two groups, although the length of hospital stay was significantly shorter for the inpatient palliative care group $(10.6 \pm 11.1$ days vs. 20.6 \pm 16.7 days, respectively; $P<0.001$ ). After one-to-one propensity score matching, 276 matched controls were selected, and we found that the baseline patient characteristics in the two groups were similar (Table 1).

\section{Aggressive procedures}

The aggressive procedures that were used to treat the inpatient palliative care group and the matched acute hospital care group are shown in Table 2. The patients in the acute hospital care group underwent significantly more aggressive procedures, including urinary catheterization, nasogastric tube feeding, central venous catheter insertion, intensive care unit admission, endotracheal intubation, abdominal drainage, cardiopulmonary resuscitation, total parenteral nutrition, percutaneous transhepatic cholangiography and drainage, chest tapping or intubation, and defibrillation/cardioversion (all $P<0.001$ ). Only one patient in the inpatient palliative care group underwent an aggressive procedure (hemodialysis).

\section{Drug prescription patterns}

The prescription patterns for both care groups are shown in Table 3, according to their Anatomical Therapeutic Chemical classification. In the inpatient palliative care group, the most commonly prescribed medication was natural opium alkaloids (84.4\%), followed by solutions that affect electrolyte balance $(80.1 \%)$, propulsives (55.1\%), contact laxatives (52.9\%), and benzodiazepine derivatives $(48.2 \%)$. In the matched acute hospital care group, the most commonly prescribed medication was solutions that affect electrolyte balance $(97.1 \%)$, followed by solutions for parenteral nutrition $(80.8 \%)$, propulsives (72.5\%), plain sulfonamide diuretics $(71.7 \%)$, and electrolyte solutions $(66.7 \%)$. Compared to the acute hospital care group, the inpatient palliative care patients more frequently received natural opium alkaloids (84.4 \% vs. $56.5 \% ; P<0.001)$ and benzodiazepine derivatives (48.2\% vs. $26.8 \% ; P<0.001$ ), although they less frequently received cephalosporins (32.2\% vs. $60.5 \%$; $P<0.001)$ and adrenergic or dopaminergic agents (5.8 \% vs. $52.9 \% ; P<0.001)$.

\section{Medical costs}

A comparison of the medical costs for both care groups is shown in Table 4 . The combined daily medical costs were significantly lower for the inpatient palliative care group, compared to those for the acute hospital care group (adjusted mean $=107$ US [101-113] dollars/day, vs . 253 [210-308] US dollars/day, respectively; $P<0.001)$. In addition, all daily medical cost categories were significantly higher in the acute hospital care group, including diagnoses, laboratory examinations, radiologic examinations, therapies, medications, and hemodialysis (all $P<0.005)$.

\section{Discussion}

In this study, we collected comprehensive data from the NHI records of patients with terminal pancreatic cancer, and used these data to compare inpatient palliative care 
Table 1 Baseline characteristics of terminal pancreatic cancer patients according to end-of-life care

\begin{tabular}{|c|c|c|c|c|c|}
\hline \multirow{3}{*}{ Characteristics } & \multirow{3}{*}{$\begin{array}{l}\text { Inpatient palliative care } \\
\text { n (\%) }\end{array}$} & \multicolumn{4}{|c|}{ Acute hospital care } \\
\hline & & \multicolumn{2}{|c|}{ Pre-matched } & \multicolumn{2}{|c|}{ Post-matched } \\
\hline & & $\mathrm{n}(\%)$ & P & $\mathrm{n}(\%)$ & $P$ \\
\hline Number of patients & $276(100)$ & $578(100)$ & & $276(100)$ & \\
\hline Age (years; mean $\pm S D$ ) & $68.6 \pm 13.4$ & $67.5 \pm 12.8$ & 0.265 & $68.5 \pm 12.4$ & 0.953 \\
\hline Sex & & & 0.687 & & 0.535 \\
\hline Male & $173(63)$ & $354(61)$ & & $180(65)$ & \\
\hline Female & $103(37)$ & $224(39)$ & & $96(35)$ & \\
\hline Physicians' specialty & & & $<0.001$ & & $<0.001$ \\
\hline Family medicine & $108(39)$ & $38(7)$ & & $24(7)$ & \\
\hline Oncology & $69(25)$ & $174(30)$ & & $77(29)$ & \\
\hline Internal medicine & $64(23)$ & $226(39)$ & & $104(43)$ & \\
\hline General & $57(21)$ & $64(11)$ & & $28(10)$ & \\
\hline Gastroenterology & $3(1)$ & $138(24)$ & & $65(24)$ & \\
\hline Cardiology & $0(0)$ & $3(1)$ & & $2(0.7)$ & \\
\hline Chest medicine & $0(0)$ & $14(2)$ & & $8(3)$ & \\
\hline Nephrology & $1(0.4)$ & $2(0.3)$ & & $0(0)$ & \\
\hline Endocrinology & $0(0)$ & $4(0.7)$ & & $1(0.4)$ & \\
\hline Infection & $3(1)$ & $1(0.2)$ & & $0(0)$ & \\
\hline Radiation oncology & $25(9)$ & $17(3)$ & & $6(2)$ & \\
\hline Surgery & $0(0)$ & $113(20)$ & & $57(18)$ & \\
\hline Neurology & $9(3)$ & $6(1)$ & & $3(1)$ & \\
\hline Others & $1(0.4)$ & $4(0.7)$ & & $4(1)$ & \\
\hline Accreditation level of hospital & & & 0.030 & & 0.304 \\
\hline Medical centers & $161(58)$ & $316(55)$ & & $156(57)$ & \\
\hline Regional hospitals & $73(26)$ & $199(34)$ & & $87(32)$ & \\
\hline District hospitals & $42(15)$ & $63(11)$ & & $33(12)$ & \\
\hline Region of hospital & & & 0.021 & & 0.668 \\
\hline North & $171(62)$ & $401(69)$ & & $171(62)$ & \\
\hline Central & $20(7)$ & $49(8)$ & & $25(9)$ & \\
\hline South & $75(27)$ & $120(21)$ & & $74(27)$ & \\
\hline East & $10(4)$ & $8(1)$ & & $6(2)$ & \\
\hline Charlson comorbidity index (mean \pm SD) & $6.8 \pm 6.5$ & $6.7 \pm 6.5$ & 0.798 & $6.9 \pm 2.8$ & 0.564 \\
\hline Length of hospital stay (day, mean \pm SD) & $10.6 \pm 11.1$ & $20.6 \pm 16.7$ & $<0.001$ & $20.6 \pm 16.3$ & $<0.001$ \\
\hline
\end{tabular}

and acute hospital care. Our analysis revealed that the majority of patients with pancreatic cancer were men and had been hospitalized in medical centers. Furthermore, most patients were treated in acute hospital wards. In the inpatient palliative care group, approximately $40 \%$ of patients were treated by family physicians, and these patients had shorter hospital stays, fewer aggressive procedures, and lower medical costs. Furthermore, these patients were prescribed more natural opium alkaloids and benzodiazepine derivatives, compared to the acute hospital care inpatients. However, given the relatively short hospital stays, we could not perform a detailed analysis of the durations for all medical procedures.

In Taiwan, the only available forms of hospice care are inpatient palliative care and home palliative care [17], and there were 50 hospital-based inpatient palliative care units and 69 home palliative care teams in April 2015 [18]. The palliative care teams only offer consultations for patients in acute hospital wards. The physicians in acute hospital wards are responsible for all care-related decisions for patients in acute hospital wards. It has been estimated that 13,000 patients with cancer receive 
Table 2 Aggressive procedures used to treat terminal pancreatic cancer patients according to end-of-life care

\begin{tabular}{|c|c|c|c|c|c|}
\hline \multirow[t]{3}{*}{ Procedures } & \multicolumn{2}{|c|}{ Inpatient palliative care } & \multicolumn{2}{|c|}{ Acute hospital care } & \multirow[t]{3}{*}{$P$} \\
\hline & \multicolumn{2}{|c|}{$(n=276)$} & \multicolumn{2}{|c|}{$(n=276)$} & \\
\hline & $\mathrm{n}$ & $\%$ & $\mathrm{n}$ & $\%$ & \\
\hline Urinary catheterization & 0 & 0 & 156 & 56.5 & $<0.001$ \\
\hline Nasogastric tube feeding & 0 & 0 & 86 & 31.2 & $<0.001$ \\
\hline Central venous catheter insertion & 0 & 0 & 77 & 27.9 & $<0.001$ \\
\hline Intensive care unit admission & 0 & 0 & 57 & 20.7 & $<0.001$ \\
\hline Endotracheal intubation & 0 & 0 & 49 & 17.8 & $<0.001$ \\
\hline Abdominal drainage & 0 & 0 & 33 & 12.0 & $<0.001$ \\
\hline Cardiopulmonary resuscitation & 0 & 0 & 28 & 10.1 & $<0.001$ \\
\hline Total parenteral nutrition & 0 & 0 & 25 & 9.1 & $<0.001$ \\
\hline Percutaneous transhepatic cholangiography and drainage & 0 & 0 & 23 & 8.3 & $<0.001$ \\
\hline Chest tapping or intubation & 0 & 0 & 15 & 5.4 & $<0.001$ \\
\hline Defibrillation/cardioversion & 0 & 0 & 9 & 3.3 & $<0.001$ \\
\hline Hemodialysis & 1 & 0.4 & 7 & 2.5 & 0.068 \\
\hline Epinephrine injection & 0 & 0 & 4 & 1.4 & 0.124 \\
\hline
\end{tabular}

these services each year in Taiwan [19], and inpatient palliative care is available and fully accessible to all healthcare beneficiaries, to whom it is offered at all medical centers and in select regional hospitals, although it is rarely offered in district hospitals. Furthermore, Taiwanese patients with pancreatic cancer are frequently referred to medical centers, which have a full complement of diagnostic facilities and greater treatment capabilities [20]. These patients can then be transferred to the inpatient palliative care units or acute hospital wards when they become terminally ill. Although sophisticated medical services are rarely used for patients who receive inpatient palliative care or acute hospital care, it is more convenient for patients at medical centers to access these services, compared to inpatients at regional or district hospital. Therefore, patients with terminal pancreatic cancer are more frequently admitted to medical centers, rather than to regional or district hospitals.

Inpatient palliative care is not widely used in Taiwan, and we found that the majority of terminally ill patients with pancreatic cancer elected to receive end-of-life care at acute hospital wards. Several factors might influence the decision to not select inpatient palliative care, such as physician preferences and referral practices, cultural concerns, individual patient choices and circumstances, and public or professional awareness of the benefits of inpatient palliative care $[9,10,21,22]$. Unfortunately, in Taiwan, inpatient palliative care units are associated with a negative image (i.e., 'death wards'), and a strong sense of familial obligation leads families to provide in-home care to sick family members. In addition, caregivers and family members typically prefer life-sustaining treatment

Table 3 Medications used to treat terminal pancreatic patients according to end-of-life care

\begin{tabular}{|c|c|c|c|c|c|c|}
\hline \multirow[t]{2}{*}{ Ranking } & \multicolumn{3}{|l|}{ Inpatient palliative care $(n=276)$} & \multicolumn{3}{|l|}{ Acute hospital care $(n=276)$} \\
\hline & Drug class (ATC code) & $\mathrm{n}$ & $\%$ & Drug class (ATC code) & $n$ & $\%$ \\
\hline 1 & Natural opium alkaloids (N02AA) & 233 & 84.4 & Solutions affecting the electrolyte balance (B05BB) & 268 & 97.1 \\
\hline 2 & Solutions affecting the electrolyte balance (B05BB) & 221 & 80.1 & Solutions for parenteral nutrition (B05BA) & 223 & 80.8 \\
\hline 3 & Propulsives (A03FA) & 152 & 55.1 & Propulsives (A03FA) & 200 & 72.5 \\
\hline 4 & Contact laxatives (A06AB) & 146 & 52.9 & Plain sulfonamide diuretics (C03CA) & 198 & 71.7 \\
\hline 5 & Benzodiazepine derivatives (N05CD) & 133 & 48.2 & Electrolyte solutions (B05XA) & 184 & 66.7 \\
\hline 6 & Solutions for parenteral nutrition (B05BA) & 124 & 44.9 & Contact laxatives (A06AB) & 167 & 60.5 \\
\hline 7 & Glucocorticoids (H02AB) & 123 & 44.6 & Cephalosporins (J01DA) & 167 & 60.5 \\
\hline 8 & Plain sulfonamide diuretics (C03CA) & 118 & 42.8 & Phenylpiperidine derivatives opioids (N02AB) & 159 & 57.6 \\
\hline 9 & Butyrophenone derivatives antipsychotics (N05AD) & 111 & 40.2 & Natural opium alkaloids (N02AA) & 156 & 56.5 \\
\hline 10 & Magnesium compounds (A02AA) & 108 & 39.1 & Adrenergic and dopaminergic agents (C01CA) & 146 & 52.9 \\
\hline
\end{tabular}

ATC Anatomical Therapeutic Chemical 
Table 4 Medical costs for terminal pancreatic cancer patients according to end-of-life care

\begin{tabular}{|c|c|c|c|c|c|}
\hline \multirow[t]{2}{*}{ Items } & \multicolumn{2}{|c|}{ Inpatient palliative care $(N=276)$} & \multicolumn{2}{|c|}{ Acute hospital care $(N=276)$} & \multirow[t]{2}{*}{$P^{*}$} \\
\hline & Adjusted mean & $95 \% \mathrm{Cl}$ & Adjusted mean & $95 \% \mathrm{Cl}$ & \\
\hline Diagnosis costs & 11.5 & $11.2-12.0$ & 12.9 & $12.3-13.5$ & 0.001 \\
\hline Laboratory examination costs & 5.9 & $4.9-6.9$ & 26.8 & $22.8-33.4$ & $<0.001$ \\
\hline Radiologic examination costs & 1.1 & $0.7-1.6$ & 16.9 & $9.6-28.0$ & $<0.001$ \\
\hline Therapeutic costs & 16.7 & $15.0-18.4$ & 25.7 & $22.1-29.9$ & $<0.001$ \\
\hline Medication costs & 25.4 & $22.4-29.6$ & 61.8 & $54.8-68.9$ & $<0.001$ \\
\hline Hemodialysis costs & 2.5 & $1.2-4.2$ & 22.7 & $12.2-38.3$ & 0.005 \\
\hline Total medical costs & 107 & $101-113$ & 253 & $210-308$ & $<0.001$ \\
\hline
\end{tabular}

All values are reported as US dollars/day

*Analyzed using a generalized linear model with gamma distribution and log link function. 95 \% Cl: 95 \% confidence interval

for terminally ill patients, and some physicians prefer not to discuss end-of-life issues with their patients [23]. Therefore, these factors may motivate Taiwanese patients and their families to elect for end-of-life care in acute hospital wards, rather than in inpatient palliative care units.

The basic philosophical tenets of end-of-life care have been rooted in the recognition of an individual's personal dignity. Therefore, the most common treatment objectives for inpatient palliative care include helping patients die with dignity, alleviating pain and suffering, controlling symptoms, and using less aggressive therapies $[10,24,25]$. In this study, we found that $84.4 \%$ of the inpatient palliative care patients were prescribed natural opium alkaloids, which is noticeably higher than the prescription rate $(72.7 \%)$ in a previous study of inpatient palliative care for patients with hepatocellular carcinoma [17]. Furthermore, only $56.5 \%$ of the patients in the acute hospital care group were prescribed natural opium alkaloids. However, among patients with pancreatic cancer, pain is a major source of distress [2], and adequate pain control is the primary priority in terminally ill cases, which may partially explain why the patients in the inpatient palliative care group more frequently received benzodiazepine derivatives $(48.2 \%$ vs. $26.8 \%$ in the acute hospital care group). These drugs are an important adjuvant to control pain, and can help treat concomitant psychological disturbances, such as insomnia, anxiety, and depression, according to the World Health Organization's guide for cancer pain relief [26]. Moreover, the patients in the inpatient palliative care group used fewer cephalosporins (32.2\% vs. $60.5 \%$, respectively) and adrenergic or dopaminergic agents $(5.8 \%$ vs. $52.9 \%$, respectively), which is likely because these treatments are typically futile in patients with terminal pancreatic cancer [27].

There were 4,686 deaths due to pancreatic cancer from 2003 to 2006 [28]. However, home palliative care is widely used in Taiwan [18], and many patients were discharged, against their physician's advice, when they were dying [29]. Thus, only 854 patients with terminal pancreatic cancer who died in-hospital were included in this study. Nevertheless, our findings indicated that there were significant differences between inpatient palliative care and acute hospital care for patients with terminal pancreatic cancer.

Although we found that inpatient palliative care resulted in significantly shorter hospital stays, there is controversy in the existing literature regarding whether inpatient palliative care leads to shorter or longer hospital stays $[7,11]$. In our study, patients in the palliative care units used fewer aggressive procedures, which may lead to shorter lifespans and shorter hospital stays. However, we also found that inpatient palliative care resulted in lower per-person or daily medical costs, compared to acute hospital care. These findings may be attributable to the patients' poor general conditions after termination of anticancer treatment, and their rapidly growing pancreatic tumors. Moreover, the treatment of patients' poor general condition and symptoms of pancreatic cancer are challenging, and typically empirical, in inpatient palliative care units. Therefore, increasing the use of solutions that affect electrolyte balance, solutions for parenteral nutrition, propulsives, electrolyte solutions, cephalosporins, and adrenergic or dopaminergic agents may prolong the lives of some patients. However, prolonging the lives of terminally ill patients, such as the patients in our study, will inevitably prolong their suffering. Therefore, as we found that inpatient palliative care cost less than acute hospital care, the cost-benefit ratio of acute hospital care should be subjected to further evaluation $[25,30,31]$. Furthermore, we only evaluated patients who were treated during 2003-2006, and the number of Taiwanese inpatient palliative care units has increased from 26 in 2004 to 53 in 2015 [32]. Therefore, inpatient palliative care has become more accepted by the general public, which further supports its consideration during end-of-life decision-making. 
The major strength of this study was its nationwide population-based design, which included a relatively large number of patients with pancreatic cancer. Furthermore, this design facilitated a comprehensive evaluation of the medical behaviors and costs that were associated with inpatient palliative care and acute hospital care. Therefore, the findings of our study provide epidemiological evidence that inpatient palliative care provides a greater amount of palliative care for patients with terminally ill pancreatic cancer and is less expensive than acute hospital care. Thus, these findings may provide the basis for changing traditional Taiwanese perceptions regarding inpatient palliative care, and for promoting end-of-life inpatient palliative care for patients with pancreatic cancer.

This study has several limitations that should be considered when interpreting our findings. First, we could not obtain data regarding the patients' educational and socioeconomic status, the preferences of the patients and their caregivers, patient life expectancies, and the attitudes of physicians toward inpatient palliative care. However, although only a limited number of covariates were included in the logistic regression model, our propensity-score matching at the patient level provided comparable baseline characteristics between the two groups, which may have eliminated some selection biases. Second, although fewer aggressive procedures and a lower cost burden are desirable factors, such as those observed in the inpatient palliative care group, we were unable to obtain data regarding the patients' symptom burdens, pain scores, or quality of life measures. Therefore, future research should incorporate quality of life measures to advance our knowledge regarding the effects of inpatient palliative care. Third, eligible patients receive a fixed daily payment from Taiwan NHI for inpatient palliative care [33], and this payment may influence the observed differences in the use of medications, aggressive procedures, and incurred costs between the two groups.

\section{Conclusions}

Inpatient palliative care units provided patients with pancreatic cancer more frequent pain control treatments, fewer aggressive procedures, and lower medical costs, compared to patients in acute hospital wards. Therefore, inpatient palliative care should be considered a viable option for treating patients with terminal pancreatic cancer.

\section{Abbreviations \\ NHI: National Health Insurance; ICD-9-CM: The International Classification of Diseases, 9th Revision, Clinical Modification; SD: standard deviation; \\ ATC: Anatomical Therapeutic Chemical; $95 \%$ Cl: $95 \%$ confidence interval.}

\section{Competing interests}

The authors declare that they have no competing interests.

\section{Authors' contributions}

All authors fulfilled the criteria for authorship. JPW, CYW, SJH, and CPL conducted the conception and design of the study. SJH and CPL obtained the data. JPW, CYW, YPH, and CPL developed the methodology. IHH and CHK conducted the data analysis and interpretation, which was supervised by SJH and CPL. JPW, CYW, and CPL provided the greatest contributions to writing the manuscript, although all authors took part in the data interpretation and drafting of the manuscript. All authors read and approved the final manuscript. CPL had full access to all of the study data, and takes responsibility for the integrity of the data and the accuracy of the analysis.

\section{Acknowledgements}

Data from this study were obtained from the National Health Insurance Research database, which is overseen by the National Health Insurance Administration, Ministry of Health and Welfare, and is managed by the National Health Research Institutes in Taiwan. The interpretations and conclusions that are contained herein do not represent those of National Health Insurance Administration, Ministry of Health and Welfare, or National Health Research Institutes. This work was supported by grants from Taipei Veterans General Hospital (V105C-169), the National Science Council (NSC 98-2314-B-075-029), the National Research Program for Biopharmaceuticals of Taiwan (100CT202), and 'Aim for the Top University Plan' of National Yang-Ming University and Ministry of Education, Taiwan. We thank the Clinical Research Core Laboratory and the Medical Science \& Technology Building of Taipei Veterans General Hospital for providing experimental space and facilities. This work was assisted in part by the Division of Experimental Surgery of the Department of Surgery, Taipei Veterans General Hospital. We also thank Editage for English editing.

\section{Author details}

'Division of Gastroenterology and Hepatology, Department of Medicine, Taipei Veterans General Hospital, No. 201, Sec. 2, Shih-Pai Road, Taipei 11217, Taiwan. ${ }^{2}$ National Yang-Ming University School of Medicine, Taipei, Taiwan. ${ }^{3}$ Department of Internal Medicine, Taipei City Hospital, Renai Branch, Taipei, Taiwan. ${ }^{4}$ Department of Dermatology, Taipei Veterans General Hospital, No. 201, Sec. 2, Shih-Pai Road, Taipei 11217, Taiwan. ${ }^{5}$ Institute of Public Health \& Department of Public Health, National Yang-Ming University, Taipei, Taiwan. ${ }^{6}$ College of Healthcare Administration and Management, National Taipei University of Nursing and Health Sciences, Taipei, Taiwan. ${ }^{7}$ Department of Oncology, Taipei Veterans General Hospital, Taipei, Taiwan. ${ }^{8}$ Department of Family Medicine, Taipei Veterans General Hospital, Taipei, Taiwan.

Received: 12 April 2015 Accepted: 6 January 2016

Published online: 08 January 2016

\section{References}

1. Siegel R, Ma J, Zou Z, Jemal A. Cancer statistics, 2014. CA Cancer J Clin. 2014;64(1):9-29.

2. Chao Y, Wu CY, Wang JP, Lee RC, Lee WP, Li CP. A randomized controlled trial of gemcitabine plus cisplatin versus gemcitabine alone in the treatment of metastatic pancreatic cancer. Cancer Chemother Pharmacol. 2013:72(3):637-42

3. Clark D, editor. Cicely Saunders - Founder of the hospice movement : Selected letters 1959-1999. London: Oxford University Press; 2005.

4. http://www.medicinenet.com/script/main/artasp?articlekey=5598. (Last accessed Nov. 8 2015.).

5. Kane RL, Wales J, Bernstein L, Leibowitz A, Kaplan S. A randomised controlled trial of hospice care. Lancet. 1984;1 (8382):890-4.

6. http://www.who.int/cancer/palliative/definition/en/. (Last accessed Nov. 8 2015.).

7. Connor SR, Pyenson B, Fitch K, Spence C, Iwasaki K. Comparing hospice and nonhospice patient survival among patients who die within a three-year window. J Pain Symptom Manage. 2007;33(3):238-46.

8. Tang ST, Chen ML, Huang EW, Koong SL, Lin GL, Hsiao SC. Hospice utilization in Taiwan by cancer patients who died between 2000 and 2004. J Pain Symptom Manage. 2007;33(4):446-53.

9. Tang ST, Huang EW, Liu TW, Wang HM, Chen JS. A population-based study on the determinants of hospice utilization in the last year of life for Taiwanese cancer decedents, 2001-2006. Psychooncology. 2010;19(11):1213-20.

10. Tang ST, Liu TW, Lai MS, McCorkle R. Discrepancy in the preferences of place of death between terminally ill cancer patients and their primary family caregivers in Taiwan. Soc Sci Med. 2005;61(7):1560-6. 
11. Chiou JD, Chen CW, Lin MH, Wu PY, Liu JY, Chen TJ, et al. A five-year analysis of the inpatient service of the hospice and palliative care unit in VGH-Taipei, Taiwan. J Fam Med. 2005;15:123-33.

12. National Health Research Institutes. National Health Insurance Research Database. http://w3.nhri.org.tw/nhird//en/Data_Subsets.html. (Last accessed Nov. 8 2015.).

13. Bureau of National Health Insurance. Medical Material Items of National Health Insurance. www.nhi.gov.tw/webdata/webdata. aspx?menu=18\&menu_id $=683 \& W D \_I D=757 \&$ webdata_id $=1166$. (Last accessed Nov. 8 2015.).

14. Charlson ME, Pompei P, Ales KL, Mackenzie CR. A new method of classifying prognostic comorbidity in longitudinal studies: development and validation. J Chronic Dis. 1987;40(5):373-83.

15. D'Agostino Jr RB. Propensity score methods for bias reduction in the comparison of a treatment to a non-randomized control group. Stat Med. 1998;17(19):2265-81.

16. Nelder J, Wedderburn R. Generalized linear models. J R Stat Soc A Stat. 1972;135:370-84

17. Hwang SJ, Chang HT, Hwang $\mathrm{IH}$, Wu CY, Yang WH, Li CP. Hospice offers more palliative care but costs less than usual care for terminal geriatric hepatocellular carcinoma patients: a nationwide study. J Palliat Med. 2013; 16(7):780-5.

18. National Health Insurance Administration. Hospice Care. http://www.nhi.gov. tw/webdata/webdata.aspx?menu=20\&menu_id=712\&webdata_ id =3650\&WD_ID=902. (Last accessed Nov. 8 2015.).

19. Ministry of Foreign Affairs. Improving Taiwan's End-of-Life Care. http:// taiwantoday.tw/ct.asp?xltem=173667\&ctNode=428. (Last accessed Nov. 8 2015.).

20. Huo TI, Lin HC, Hsia CY, Wu JC, Lee PC, Chi CW, et al. The model for endstage liver disease based cancer staging systems are better prognostic models for hepatocellular carcinoma: a prospective sequential survey. Am J Gastroenterol. 2007;102(9):1920-30.

21. Beccaro M, Costantini M, Giorgi Rossi P, Miccinesi G, Grimaldi M, Bruzzi P, et al. Actual and preferred place of death of cancer patients. Results from the Italian survey of the dying of cancer (ISDOC). J Epidemiol Community Health. 2006;60(5):412-6.

22. Higginson IJ, Sen-Gupta GJ. Place of care in advanced cancer: a qualitative systematic literature review of patient preferences. J Palliat Med. 2000;3(3): 287-300.

23. Keating NL, Landrum MB, Rogers Jr SO, Baum SK, Virnig BA, Huskamp HA, et al. Physician factors associated with discussions about end-of-life care. Cancer. 2010;116(4):998-1006.

24. Loke SS, Rau KM. Differences between inpatient hospice care and in-hospital nonhospice care for cancer patients. Cancer Nurs. 2011;34(3):E21-26.

25. Lin WY, Chiu TY, Hsu HS, Davidson LE, Lin T, Cheng KC, et al. Medical expenditure and family satisfaction between hospice and general care in terminal cancer patients in Taiwan. J Formos Med Assoc. 2009;108(10):794-802.

26. World Health Organization. Cancer pain relief. 2nd ed. Geneva: WHO; 1996.

27. Ramondetta $L$. What is the appropriate approach to treating women with incurable cervical cancer? J Natl Compr Canc Netw. 2013:11(3):348-55.

28. Taiwan Cancer Registry. http://tcr.cph.ntu.edu.tw/main.php?Page=N2. (Last accessed Nov. 8 2015.)

29. Tang ST. Meanings of dying at home for Chinese patients in Taiwan with terminal cancer: a literature review. Cancer Nurs. 2000;23(5):367-70.

30. Lo JC. The impact of hospices on health care expenditures-the case of Taiwan. Soc Sci Med. 2002;54(6):981-91.

31. Pyenson B, Connor S, Fitch K, Kinzbrunner B. Medicare cost in matched hospice and non-hospice cohorts. J Pain Symptom Manage. 2004;28(3):200-10.

32. Hospice and palliative care in Taiwan. https:/www.ehospice.com/Default/ tabid/10686/Articleld/15164/. (Last accessed Nov. 8 2015.).

33. Bureau of National Health Insurance. http:/www.nhi.gov.tw/epaperN/temDetail. aspx?DatalD=2963\&lsWebData=0\&ltemTypelD=5\&Papers|D=252\&PiclD=. (Last accessed Nov. 8 2015.)

\section{Submit your next manuscript to BioMed Central and we will help you at every step:}

- We accept pre-submission inquiries

- Our selector tool helps you to find the most relevant journal

- We provide round the clock customer support

- Convenient online submission

- Thorough peer review

- Inclusion in PubMed and all major indexing services

- Maximum visibility for your research

Submit your manuscript at www.biomedcentral.com/submit

) Biomed Central 